\title{
Global miRNA expression is temporally correlated with acute kidney injury in mice
}

Rui Cui, Jia Xu, Xiao Chen, Wenliang Zhu

MicroRNAs (miRNAs) are negative regulators of gene expression and protein abundance. Current evidence shows an association of miRNAs with acute kidney injury (AKI) leading to substantially increased morbidity and mortality. Here, we investigated whether miRNAs are inductive regulators responsible for the pathological development of AKI. Microarray analysis was used to detect temporal changes in global miRNA expression within $48 \mathrm{~h}$ after AKI in mice. Results indicated that global miRNA expression gradually increased over $24 \mathrm{~h}$ from ischemia reperfusion injury after $24 \mathrm{~h}$, and then decreased from $24 \mathrm{~h}$ to $48 \mathrm{~h}$. A similar trend was observed for the index of tubulointerstitial injury and the level of serum creatinine, and there was a significant correlation between the level of total miRNA expression and the level of serum creatinine $(p<0.05)$. This expression-phenotype correlation was validated by quantitative reverse transcription PCR on individual miRNAs, including miR-18a, -134, -182, -210 and -214. Increased global miRNA expression may lead to widespread translational repression and reduced cellular activity. Furthermore, significant inflammatory cytokine release and peritubular capillary loss were observed, suggesting that the initiation of systematic destruction programs was due to AKI. Our findings provide new understanding of the dominant role of miRNAs in promoting the pathological development of AKI. 
1 Global miRNA expression is temporally correlated with acute kidney injury

3 Rui Cui ${ }^{1, *}$, Jia Xu ${ }^{1, *}$, Xiao Chen ${ }^{2}$, Wenliang $\mathrm{Zhu}^{3 \#}$

${ }^{1}$ Department of Nephrology, the Fourth Affiliated Hospital of Harbin Medical University, Harbin, China

$5 \quad{ }^{2}$ Department of Nephrology, Heilongjiang Province Hospital, Harbin, China

$6 \quad{ }^{3}$ Institute of Clinical Pharmacology, the Second Affiliated Hospital of Harbin Medical University, Harbin,

7 China

*These authors contributed equally to this work.

\#Corresponding authors: Wenliang Zhu, Phone/Fax: 86-451-8629-773, E-mail: wenzwl@yeah.net. 
22 MicroRNAs (miRNAs) are negative regulators of gene expression and protein abundance. Current evidence

23 shows an association of miRNAs with acute kidney injury (AKI) leading to substantially increased

24 morbidity and mortality. Here, we investigated whether miRNAs are inductive regulators responsible for

25 the pathological development of AKI. Microarray analysis was used to detect temporal changes in global miRNA expression within $48 \mathrm{~h}$ after AKI in mice. Results indicated that global miRNA expression gradually increased over $24 \mathrm{~h}$ from ischemia reperfusion injury after $24 \mathrm{~h}$, and then decreased from $24 \mathrm{~h}$ to $48 \mathrm{~h}$. A similar trend was observed for the index of tubulointerstitial injury and the level of serum creatinine, and there was a significant correlation between the level of total miRNA expression and the level of serum creatinine $(p<0.05)$. This expression-phenotype correlation was validated by quantitative reverse transcription PCR on individual miRNAs, including miR-18a, -134, -182, -210 and -214. Increased global miRNA expression may lead to widespread translational repression and reduced cellular activity. Furthermore, significant inflammatory cytokine release and peritubular capillary loss were observed, suggesting that the initiation of systematic destruction programs was due to AKI. Our findings provide new understanding of the dominant role of miRNAs in promoting the pathological development of AKI.

\section{Introduction}

Acute kidney injury (AKI) is a common clinical syndrome mainly characterized by a rapid decline in kidney 
43 function (Srisawat and Kellum, 2011). The leading etiology is ischemia reperfusion injury (IRI), followed

44 by sepsis and nephrotoxic insult (Chertow et al., 2005; Mehta et al., 2007; Waikar et al., 2008). IRI is caused

by an abrupt, transient decline in blood flow to the kidney (Lien et al., 2003) and leads to the production

and release of inflammatory cytokines, infiltration of neutrophils and macrophages, and loss of peritubular

capillaries (PTC; Ishii et al., 2005; Furuichi et al., 2009). It was suggested that IRI-induced PTC loss was

directly associated with reduced kidney function and the progression to renal fibrosis (Li, et al., 2010).

Despite advances in clinical care, patients with AKI continue to have significant morbidity and mortality

(Aydin et al., 2007). Although many therapeutic strategies have been proposed, few have been proven to

effectively improve AKI, making this disease a vexing clinical problem worldwide.

MicroRNAs (miRNAs) are a superclass of endogenous small non-coding RNAs ( 22 nucleotides). With

the exception of miR-373 (Place et al., 2008), the vast majority of miRNAs can be defined as negative

regulators of gene expression at the post-transcriptional level (Bartel, 2004). Currently, there are more than

2800 known unique mature miRNAs transcribed by the human genome (Kozomara and Griffiths-Jones,

2014). Approximately two-thirds of all human mRNA genes are affected by miRNA-mediated translational

repression. Researchers generally agree on the high potential of miRNAs as therapeutic targets and

diagnostic markers, as miRNAs are involved in nearly all human diseases, playing important roles in

regulating pathologically related genes (Asli et al., 2008). miRNAs are involved in various biological

processes, such as hypoxia, inflammation, cell death, and fibrosis, indicating indispensable roles in renal

pathophysiology (Saal and Harvey, 2009; Bhatt et al., 2011; Chung et al., 2013; Wei et al., 2013; Ma and

$\mathrm{Qu}, 2013)$. 
programs, such as apoptotic and inflammatory pathways, and eventually reducing kidney function (Basile et al., 2012). In contrast, the reparative processes associated with AKI are inefficient. Thus, imbalance between injury and repair results in damaged kidney function and structure, eventually leading to renal fibrosis and progression to chronic kidney disease (Coca et al., 2012). Here, we hypothesized that negative regulators of gene expression such as miRNAs may be critical repressors of cellular activities during AKI, when energy production and other cellular activities are significantly reduced. This hypothesis was supported by recent evidence that miRNAs negatively regulated the cell cycle in AKI (Khalid et al., 2014).

Thus, miRNAs were suggested to be important initiators that contribute to the imbalance between injury and repair in AKI.

To test this hypothesis, a renal IRI mouse model was established and microarray analysis was used to detect changes in temporal expression of global miRNAs within $48 \mathrm{~h}$ after AKI. A potential correlation between the level of miRNA expression and kidney injury indexes (index of tubulointerstitial injury and level of serum creatinine) was tested. Additionally, temporal changes in inflammatory cytokine release and PTC loss were also determined. Together, these studies aimed to explore the role of miRNAs in promoting AKI-mediated decline in kidney function.

\section{Materials and Methods}

\section{Animals and animal models}

The use of vertebrate animals was approved by the Experimental Animal Ethic Committee of the Harbin Medical University, China (Animal Experimental Ethical Inspection Protocol, No. 2009104) and followed the Guide for the Care and Use of Laboratory Animals published by the U.S. National Institutes of Health ( $8^{\text {th }}$ Edition, 2011). Healthy male C57B/6 mice (20-25 g) were maintained under standard animal room 
conditions (temperature $21 \pm 1^{\circ} \mathrm{C}$; humidity, 55-60\%), with food and water, ad libitum, for 1 week before in vivo experiments. Animals were subjected to renal bilateral IRI using methods described previously (Lin et al., 2010). The warm ischemic time was $30 \mathrm{~min}$. Sham-operated mice, which underwent the same surgical procedure without placement of the vascular clamp, served as controls (sham group). Mice were sacrificed at $6,12,24$, and $48 \mathrm{~h}$ after reperfusion ( $n=8$ per time point). Plasma samples were taken from the tail vein to analyze the creatinine level using methods previously described (Lin et al., 2010). The mice were perfused with ice-cold normal saline via the left ventricle for $2 \mathrm{~min}$, and the kidney was rapidly excised.

Tissues were divided for histopathological analysis and molecular biological measurements, and portions of the kidney were fixed or snap-frozen in liquid nitrogen until analysis.

\section{Histological analysis and immunostaining}

As described previously (Lin et al., 2010), a section of each kidney tissue sample was embedded in paraffin (stored at $4-8^{\circ} \mathrm{C}$ ), and another section was embedded in Tissue-Tek O.C.T. compound (Sakura Finetek, Torrance, CA, USA) and then stored at $-80^{\circ} \mathrm{C}$. Frozen sections were obtained using a cryostat (Thermo Scientific, Cheshire, UK) at $4 \mu \mathrm{m}$, and paraffin sections were obtained using a microtome (Thermo Scientific, Walldorf, Germany) set at 2-3 $\mu \mathrm{m}$. Paraffin sections were stained with hematoxylin-eosin (HE) and Masson's trichrome using standard techniques. Immunofluorescence labeling was performed using a previously described protocol (Peng et al., 2014). Primary antibodies against the following proteins were used for immunolabeling: rat anti-mouse F4/80 (1:200, clone BM8; eBioscience, San Diego, CA, USA), and rabbit anti-mouse CD31 (1:50, Abcam, Hong Kong). The secondary antibodies were Alexa Fluor 488conjugated goat anti-rabbit and Alexa Fluor 488-conjugated goat anti-rat (1:200; Jackson ImmunoResearch Laboratories, West Grove, PA, USA). Nuclei were stained using 4,6-diamidino-2-phenylindole (DAPI). 
Images were captured using a Nikon microscope (Tokyo, Japan) and processed using NIS-Elements software (Tokyo, Japan).

All tissue sections were analyzed using 10-15 random outer medulla of kidney per mouse. The data obtained from each tissue are represented by the mean of all fields. The degree of tubulointerstitial injury in HE-stained paraffin sections and F4/80-positive cells were determined as previously described (Cui et al., 2014). CD31-labeled kidney sections were used to evaluate PTC loss as follows: each image was divided into 252 squares, where one square without a PTC was considered positive for loss, and the final score was presented as the percentage of positive squares.

\section{Enzyme-linked immunosorbent assay (ELISA)}

Mouse TNF- $\alpha$, IL-1 $\beta$, and IL-6 ELISA kits (R\&D Systems, Minneapolis, MN, USA) were used to determine the concentrations of these markers in kidney tissue homogenates. Briefly, a total volume of 50 $\mu \mathrm{L}$, containing $10 \mu \mathrm{L}$ tissue homogenate and $40 \mu \mathrm{L}$ sample diluent, was added to each sample well. Horseradish peroxidase-conjugated reagent was added to each well and incubated for $1 \mathrm{~h}$ at $37^{\circ} \mathrm{C}$.

Tetramethylbenzidine was added after the array was washed. The chromogenic reaction was terminated with stop solution, and the absorbance was measured at $450 \mathrm{~nm}$ with an iMark Microplate Reader (BIORAD, Tokyo, Japan). The BCA Protein Assay Kit (Beyotime Biotechnology Co., Ltd., Shanghai, China) was used to determine the total protein concentration of the kidney tissue homogenates.

\section{Tissue sample collection and total RNA extraction}

Total RNA was extracted from $20 \mathrm{mg}$ of frozen renal tissue using Trizol reagent (Invitrogen, Shanghai, China), and stored at $-80^{\circ} \mathrm{C}$. The RNA eluate was stored at $-80^{\circ} \mathrm{C}$. All RNA extraction samples were thawed and then restored at $-80^{\circ} \mathrm{C}$ for further experiments. 


\section{Microarray assay}

Oebiotech Technology Co, Ltd. (Shanghai, China) performed the miRNA microarray assay. Global miRNA expression was detected in the tissue samples using the Agilent mouse miRNA microarray (Release 19.0, $8 \times 60 \mathrm{~K})$. Sample labeling, microarray hybridization, and array washes were performed according to manufacturer standard protocols (Agilent Technologies Inc., Santa Clara, CA, USA) as previously described (Meng et al., 2015). The microarray data were submitted to the Gene Expression Omnibus (GEO) database (accession number: GSE75076). For a single miRNA, miRNA abundance was defined as the raw microarray signal output normalized to the blank value 0.1 . Total miRNA abundance was defined as the sum of the miRNA abundance values for all miRNAs $(n=1247)$ detected by microarray.

\section{KEGG pathway analysis}

Based on the microarray data, a pathway analysis on KEGG (Kanehisa et al., 2016) was performed to investigate whether some pathways were significantly regulated by miRNAs in mouse renal pathology. Briefly, experimentally validated miRNA-target genes in mice were retrieved from the miRNA-target interaction database miRTarbase (Release 6.0; Chou et al., 2016). The online tool Gene Prospector (Yu et al., 2008) was then used to obtain genes implicated in renal pathology by using 'kidney disease' as the search term. The bioinformatics software Cytoscape v2.8.3 (Smoot et al., 2011) was applied to identify associations between miRNAs and renal pathology-related mRNA genes. The DAVID functional annotation tool (Huang et al., 2009) was used to investigate whether some KEGG signaling pathways were significantly regulated by miRNAs in renal pathology when all the renal pathology-related miRNA target genes were uploaded as a gene list and the false discovery rate (FDR) was set at 0.05 . Notably, only the miRNAs that were detected as presenting non-null microarray expression in least two time points were 
148 included in the analysis.

149 Quantitative reverse transcription-polymerase chain reaction (qRT-PCR)

150 qRT-PCR was used to assess the temporal expression changes of six miRNAs including miR-18a, $-34 \mathrm{~b},-$

$151134,-182,-210$, and -214 . Briefly, $0.4 \mu \mathrm{L}$ total RNA was reverse transcribed into cDNA in $10 \mu \mathrm{L}$ reactions

152 using a high-capacity cDNA reverse transcription kit (Applied Biosystems, Foster City, CA, USA). The

cDNA was diluted 100 -fold and assayed by PCR in a $10 \mu \mathrm{L}$ reaction volume. The sequences of primers

used for amplification of miRNAs and the internal control, U6, are shown in Table S1. For qRT-PCR,

2×SYBR Green PCR Master Mix (Applied Biosystems, Warrington, UK) was used according to

manufacturer instructions with a Bio-Rad CFX96 Touch Real-Time PCR Detection System (Singapore).

After a brief denaturation cycle $\left(10 \mathrm{~min}\right.$ at $\left.95^{\circ} \mathrm{C}\right)$, amplification parameters were as follows: $95^{\circ} \mathrm{C}$ for $15 \mathrm{~s}$

and $60^{\circ} \mathrm{C}$ for $1 \mathrm{~min}$ for 40 cycles. Cycle threshold (CT) values of miRNAs were normalized to U6, and fold

change was calculated using the equation $2^{-\Delta \Delta \mathrm{CT}}$ as previously described (Wang et al., 2012).

160

161

162

163

164

165

166

167

\section{Statistical analysis}

All data are presented as the mean \pm standard error of mean (SEM). Statistical analyses were performed using one-way ANOVA, Kruskal-Wallis, and Pearson correlation tests using GraphPad Prism v6.0 (GraphPad Software, Inc., La Jolla, CA, USA). A $p<0.05$ was considered statistically significant.

\section{Results}

\section{AKI induced abrupt decline in kidney function}

In the present study, an IRI mouse model was established by renal bilateral IRI surgery. The mouse kidneys showed substantial injury after IRI, including tubular swelling and deformation, expansion, inflammatory 
cell infiltration, tubular epithelial cell degeneration and necrosis, and accumulation of necrotic material within the lumen (Figure 1A and B). The degree of damage increased with the time of reperfusion, which peaked at $24 \mathrm{~h}$ after IRI. This pathological damage of kidney tissue decreased at $48 \mathrm{~h}$ after IRI. Consistent with this result, both the index of tubulointerstitial injury and the level of serum creatinine were found to gradually increase from $6 \mathrm{~h}$ to $24 \mathrm{~h}$ as compared to those measured in sham animals, and then decreased slightly from $24 \mathrm{~h}$ to $48 \mathrm{~h}$ (Figure $1 \mathrm{C}$ and D).

\section{Temporal miRNA expression correlates with serum creatinine level}

miRNAs were previously implicated in the pathological progression of AKI (Lee et al., 2014). In this study, we observed for the first time a global, temporal change in miRNA expression, similar to the temporal trend of substantial injury after IRI (Figures 1A and 2A). Furthermore, we investigated a potential correlation between global miRNA abundance and two indicators of kidney function (index of tubulointerstitial injury and level of serum creatinine). There was no significant correlation between total miRNA abundance and the index of tubulointerstitial injury $(\mathrm{R}=0.668, p=0.22$; Figure $2 \mathrm{~B})$. Comparably, our result indicated a significantly moderate correlation between total miRNA abundance and the level of serum creatinine $(\mathrm{R}=$ $0.710, p=0.029 ;$ Figure 2C).

Furthermore, six miRNAs (miR-18a, -34b, -134, -182, -210, and -214) were examined for a similar association between temporal expression and phenotype as was observed for global miRNAs (Figure 2B and C). qRT-PCR was used to detect temporal changes in expression for each. As shown in Figure 3A, all miRNAs showed a peak increase in expression at $24 \mathrm{~h}$ after IRI. The Pearson correlation test indicated a moderate, but insignificant degree of correlation between miRNA expression and the index of 
tubulointerstitial injury (Figure 3B). With the exception of miR-34b, there was a significant correlation between the expression of each of the miRNAs and the level of serum creatinine ( $p<0.05$; Figure 3C). Notably, a correlation coefficient $>0.9$ indicated a strong correlation between the level of serum creatinine and temporal expression of miR-134/-214.

\section{miRNAs significantly regulated multiple renal pathology-related pathways}

Among the miRNAs detected by microarray, 172 presented non-null microarray expression in least two time points and constructed 1462 experimentally validated interactions with 548 renal pathology-related mRNA genes. A KEGG pathway analysis on the 548 target genes revealed that miRNAs significantly regulated 10 renal pathology-related signaling pathways (FDR $<0.05$, Table S2). The Wnt signaling pathway was the top pathway that was significantly enriched with miRNA target genes $\left(\mathrm{FDR}=5.8 \times 10^{-5}\right)$, followed by the p53 signaling pathway $\left(\mathrm{FDR}=7.6 \times 10^{-5}\right)$ and the TGF- $\beta$ signaling pathway $(\mathrm{FDR}=$ $\left.1.0 \times 10^{-4}\right)$ ). Furthermore, we investigated whether miR-18a, $-134,-182,-210$, and -214 be involved in the 10 renal pathology-related pathways. Each of the five miRNAs was experimentally validated to present kidney expression and for interactions with renal pathology-related mRNA gene(s) (Table S3). Our pathway analysis revealed that miR-18a targeted the pten gene (phosphatase and tensin homolog) in the p53 signaling pathway, miR-210 targeted the $t c f 7 l 2$ gene (transcription factor 7-like 2) in the Wnt signaling pathway, and the $b c l 2$ gene (B-cell CLL/lymphoma 2$)$ in the apoptosis signaling pathway (FDR $=1.2 \times 10^{-2}$ ). However, based on the latest release of miRTarBase (Chou et al., 2016), no miRNA-target interactions were found for miR-134, -182 , and -214 in the 10 pathways.

\section{AKI induced release of inflammatory cytokines and PTC loss}


Macrophages were previously reported to be involved in both kidney injury and kidney repair after injury (Lee et al., 2011). In the present study, a significant increase in the number of macrophages was observed in IRI mice as compared to that observed in sham-operated mice (Figures 4A and B). Expression of inflammatory cytokine proteins, including tumor necrosis factor- $\alpha$ (TNF- $\alpha$ ), interleukin (IL)-1 $\beta$, and IL-6, significantly increased in the kidneys of mice after IRI (Figure 4C-E).

PTC loss plays a central role in kidney IRI (Li et al., 2010). Maintenance of the microvasculature appears to be critical for kidney repair and preserving kidney function. To analyze peritubular capillary regeneration, we used CD31 as a marker for endothelial cells to evaluate PTC loss. PTC loss increased in kidney tissue with ischemia and reperfusion (Figure 5A). These results are shown in Figure 5B.

\section{Discussion}

Recently, the irreplaceable roles of miRNAs in kidney diseases, such as AKI, have been highlighted (Bhatt et al., 2011; Wei et al, 2013; Ma and Qu, 2013). Studies have suggested that miRNAs affect AKI pathology by interfering with regulation of the cell cycle (Khalid et al., 2014), and that altered miRNA profiles might be directly related to renal dysfunction secondary to AKI (Kumar et al., 2014). Additionally, miRNAs were identified as potential disease biomarkers for AKI (Aguado-Fraile et al., 2013). Therefore, current evidence strongly suggests that miRNAs may be key initiation factors in the pathological progression of AKI.

The present study investigated the temporal correlation between miRNA expression and macroscopic kidney function. Two indicators, index of tubulointerstitial injury and level of serum creatinine, were chosen as phenotypic indicators of kidney function. After establishing an IRI mouse model, the two indictors and global miRNA expression levels were measured at multiple time points within $48 \mathrm{~h}$ after AKI. 
228 Our results indicated abrupt aggravation of kidney injuries caused by IRI. Microarray analysis also 229 indicated a substantial increase in global miRNA expression. This finding was consistent with a previous 230 study by Godwin et al. (2010), in which the expression of many miRNAs was significantly elevated during 231 AKI (Godwin et al., 2010). Our correlation analysis indicated that global, temporal miRNA expression significantly correlated with the level of serum creatinine but not the index of tubulointerstitial injury $(\mathrm{R}=$ 0.71 and $p<0.05$ ). Ischemia could cause injuries to multiple sites in the kidney, such as tubules and glomerulus. It was validated that miRNAs were implicated in pathological processes in the two sites, suggesting global involvement of miRNAs in AKI (Ho et al., 2008; Lu et al., 2012). Additionally, the level of serum creatinine might more accurately reflect overall renal function than the index of tubulointerstitial injury (Johnson and Johnson, 2005). Based on this finding, the index of tubulointerstitial injury should be considered a local indicator of kidney function. The significant correlation between miRNA expression and a global kidney function indicator, such as serum creatinine level, is functionally equivalent to the ubiquitous roles of miRNAs in human pathophysiology (Asli et al., 2008). In particular, we demonstrated that this correlation was true for a number of individual miRNAs. The expression of five miRNAs, including miR-18a, $-134,-182,-210$, and -214 , increased at $24 \mathrm{~h}$ after IRI. The significant correlation with the level of serum creatinine strongly implied an important role for the individual miRNAs in promoting the pathological development of AKI. Our result was in line with the work by Wilflingseder et al. (2014), in which expression correlation analysis identified miR-182 as a key regulator of post-transplant AKI (Wilflingseder et al., 2014). In another clinical study, circulating miR-210 was indicated as a survival prediction biomarker for critically ill patients with AKI (Lorenzen et al., 2011). Until now, there was no experimental evidence linking miR-18a, -134 , and -214 to AKI. In this study, the expression of each of 
249 these miRNAs was significantly elevated. It was thought that significant expression and phenotype 250 association provided a clue for disclosing vital miRNAs responsible for the pathological progression of 251 AKI.

252 Furthermore, 10 KEGG pathways were revealed to be significantly regulated by miRNAs in renal pathology, which was consistent with previous studies reporting that miRNAs are vital regulators of signal transduction in renal pathologies (Badal and Danesh, 2015; Trionfini et al., 2015). Dysregulated p53 and Wnt signaling pathways were associated with the increased level of serum creatinine aroused by renal pathology (Ghosh et al., 2015; Mo et al., 2015). Our results revealed a potential role of miR-18a in regulating the p53 signaling pathway and abnormal expression of miR-210 in AKI might influence the Wnt and apoptosis signaling pathways. Our findings underscore the need for additional functional studies of miRNAs in the future.

Because they are negative post-transcriptional regulators of gene expression, increased expression of global miRNAs may result in widespread inhibition of mRNA translation into proteins. From a cellular biology perspective, an abrupt decrease in protein translation may cause reduced cellular activity and energy production (Khalid et al., 2014). Together, these effects would inevitably lead to structural and functional injures of the kidney at the tissue level (Asli et al., 2008). Our study revealed that global miRNA expression in kidney tissue increased after IRI. The rate of the increase in miRNA expression correlated with the rate of increase in serum creatinine level. This finding suggested a causal connection between changes in global miRNA expression and damaged kidney function in AKI. We also demonstrated continuous, steady-state release of inflammatory cytokines and PTC loss in AKI. This result may be a programmed consequence of 
269

270

271

272

273

274

275

276

277

278

279

280

281

282

283

284

285

286

287

288

ischemic AKI that leads to an imbalance between injury and repair (Basile et al., 2012; Khalid et al., 2014).

It validated dual roles for macrophages in rapidly invading the injured renal tissue and then reestablishing

a developmental program contributing to kidney repair after injury (Lin et al., 2010). As our experiments

were performed within $48 \mathrm{~h}$ after acute ischemic kidney injury, the negtive effect of macrophages occupied

a dominant position, explaining the further increase of F4/80 and inflammatory cytokines at $48 \mathrm{~h}$.

AKI is a common medical syndrome found in hospitalized patients (Srisawat and Kellum, 2011). Due to

a lack of effective treatment, AKI continues to be a vexing clinical problem for medical professionals worldwide. Despite recent progress in research, the pathological mechanisms underlying the AKI initiation and progression are still not fully understood (Bonventre and Yang, 2011; Malek and Nematbakhsh, 2015).

Based on our observations and results from this study and a previous study (Khalid et al., 2014), we suggest that miRNAs act as molecular inducers of AKI progression and that altered global miRNA profiles may be responsible for pathological development of AKI. Among miRNAs, a selected few, such as miR-134 and 214 , may play critical roles in this pathological course. However, further research is needed to define the pathology-initiating role of key miRNAs in AKI. In summary, our study provided novel insight into the roles of miRNAs on the progression of AKI pathology from the aspect of temporal expression of global miRNAs.

\section{Funding}

This study was supported by research grants from the National Natural Science Foundation of China (31301095), the Funds of Health and Family Planning Commission of Heilongjiang Province (2013-110, 2014-379), and the Funds of Heilongjiang Postdoctoral Financial Assistance (LBH-Z14159, LBH-Z15165). 
289 Conflicts of interest

290 The authors declare that they have no competing interests.

\section{References}

292

1. Aguado-Fraile E, Ramos E, Conde E, Rodríguez M, Liaño F, García-Bermejo ML. 2013. MicroRNAs in the kidney: novel biomarkers of acute kidney injury. Nefrologia, 33(6): 826-834 DOI 10.3265/Nefrologia.pre2013.Aug.12198.

2. Asli NS, Pitulescu ME, Kessel M. 2008. MicroRNAs in organogenesis and disease. Curr Mol Med, 8(8): 698-710 DOI 10.2174/156652408786733739.

3. Aydin Z, van Zonneveld AJ, de Fijter JW, Rabelink TJ. 2007. New horizons in prevention and treatment of ischaemic injury to kidney transplants. Nephrol Dial Transplant, 22(2): 342-346 DOI $10.1093 / \mathrm{ndt} / \mathrm{gfl} 690$.

4. Badal SS, Danesh FR. 2015. MicroRNAs and their applications in kidney diseases. Pediatr Nephrol, 30(5): 727-740 DOI 10.1007/s00467-014-2867-7.

5. Bartel DP. 2004. MicroRNAs: genomics, biogenesis, mechanism, and function. Cell, 116(2): 281-297 DOI 10.1016/S0092-8674(04)00045-5.

6. Basile DP, Anderson MD, Sutton TA. 2012. Pathophysiology of acute kidney injury. Compr Physiol, 2(2): 1303-1353 DOI 10.1002/cphy.c110041.

7. Bhatt K, Mi QS, Dong Z. 2011. microRNAs in kidneys: biogenesis, regulation, and pathophysiological roles. Am J Physiol Renal Physiol, 300(3): F602-F610 DOI 10.1152/ajprenal.00727.2010. 
309

310

311

312

313

314

315

316

317

318

319

320

321

322

323

324

325

326

327

328

8. Bonventre JV, Yang L. 2011. Cellular pathophysiology of ischemic acute kidney injury. J Clin Invest, 121(11): 4210-4221 DOI 10.1172/JCI45161.

9. Chertow GM, Burdick E, Honour M, Bonventre JV, Bates DW. 2005. Acute kidney injury, mortality, length of stay, and costs in hospitalized patients. J Am Soc Nephrol, 16(11): 3365-3370 DOI 10.1681/ASN.2004090740.

10. Chou CH, Chang NW, Shrestha S, Hsu SD, Lin YL, Lee WH, Yang CD, Hong HC, Wei TY, Tu SJ, Tsai TR, Ho SY, Jian TY, Wu HY, Chen PR, Lin NC, Huang HT, Yang TL, Pai CY, Tai CS, Chen WL, Huang CY, Liu CC, Weng SL, Liao KW, Hsu WL, Huang HD. 2016. miRTarBase 2016: updates to the experimentally validated miRNA-target interactions database. Nucleic Acids Res, 44(D1): D239-D247 DOI 10.1093/nar/gkv1258.

11. Chung AC, Yu X, Lan HY. 2013. MicroRNA and nephropathy: emerging concepts. Int J Nephrol Renovasc Dis, 6: 169-179 DOI 10.2147/IJNRD.S37885.

12. Coca SG, Singanamala S, Parikh CR. 2012. Chronic kidney disease after acute kidney injury: a systematic review and meta-analysis. Kidney Int, 81(5): 442-448 DOI 10.1038/ki.2011.379.

13. Cui R, Chen X, Peng L, Ma J, Zhu D, Li T, Wei Q, Li B. 2014. Multiple mechanisms in renal artery stenosis-induced renal interstitial fibrosis. Nephron Exp Nephrol, 128(1-2): 57-66 DOI $10.1159 / 000366481$.

14. Furuichi K, Kaneko S, Wada T. 2009. Chemokine/chemokine receptor-mediated inflammation regulates pathologic changes from acute kidney injury to chronic kidney disease. Clin Exp Nephrol, 13(1): 9-14 DOI 10.1007/s10157-008-0119-5. 
329

330

331

332

333

334

335

336

337

338

339

340

341

342

343

344

345

346

347

348

15. Ghosh P, Singha Roy S, Basu A, Bhattacharjee A, Bhattacharya S. 2015. Sensitization of cisplatin therapy by a naphthalimide based organoselenium compound through modulation of antioxidant enzymes and p53 mediated apoptosis. Free Radic Res, 49(4): 453-471 DOI 10.3109/10715762.2015.1012079.

16. Godwin JG, Ge X, Stephan K, Jurisch A, Tullius SG, Iacomini J. 2010. Identification of a microRNA signature of renal ischemia reperfusion injury. Proc Natl Acad Sci U S A, 107(32): 1433914344 DOI 10.1073/pnas.0912701107.

17. Ho J, Ng KH, Rosen S, Dostal A, Gregory RI, Kreidberg JA. 2008. Podocyte-specific loss of functional microRNAs leads to rapid glomerular and tubular injury. $J$ Am Soc Nephrol, 19(11): 20692075 DOI 10.1681/ASN.2008020162.

18. Huang da W, Sherman BT, Lempicki RA. 2009. Systematic and integrative analysis of large gene lists using DAVID bioinformatics resources. Nat Protoc, 4(1): 44-57 DOI 10.1038/nprot.2008.211.

19. Ishii Y, Sawada T, Kubota K, Fuchinoue S, Teraoka S, Shimizu A. 2005. Injury and progressive loss of peritubular capillaries in the development of chronic allograft nephropathy. Kidney Int, 67(1): 321-332 DOI 10.1111/j.1523-1755.2005.00085.x.

20. Johnson D, Johnson D. 2005. Evaluation of renal function: Use of serum creatinine concentration to assess level of kidney function. Nephrology, 10(Supp. 4): S133-S139 DOI 10.1111/j.14401797.2005.00487_1.x.

21. Kanehisa M, Sato Y, Kawashima M, Furumichi M, Tanabe M. 2016. KEGG as a reference resource for gene and protein annotation. Nucleic Acids Res, 44(D1): D457-D462 DOI 10.1093/nar/gkv1070. 
22. Khalid U, Bowen T, Fraser DJ, Jenkins RH. 2014. Acute kidney injury: a paradigm for miRNA regulation of the cell cycle. Biochem Soc Trans, 42(4): 1219-1223 DOI 10.1042/BST20140093.

23. Kozomara A, Griffiths-Jones S. 2014. miRBase: annotating high confidence microRNAs using deep sequencing data. Nucleic Acids Res, 42(Database issue): D68-D73 DOI 10.1093/nar/gkt1181.

24. Kumar S, Liu J, McMahon AP. 2014. Defining the acute kidney injury and repair transcriptome. Semin Nephrol, 34(4): 404-417 DOI 10.1016/j.semnephrol.2014.06.007.

25. Lee CG, Kim JG, Kim HJ, Kwon HK, Cho IJ, Choi DW, Lee WH, Kim WD, Hwang SJ, Choi S, Kim SG. 2014. Discovery of an integrative network of microRNAs and transcriptomics changes for acute kidney injury. Kidney Int, 86(5): 943-953 DOI 10.1038/ki.2014.117.

26. Lee S, Huen S, Nishio H, Nishio S, Lee HK, Choi BS, Ruhrberg C, Cantley LG. 2011. Distinct macrophage phenotypes contribute to kidney injury and repair. J Am Soc Nephrol, 22(2): 317-326 DOI 10.1681/ASN.2009060615.

27. Li B, Cohen A, Hudson TE, Motlagh D, Amrani DL, Duffield JS. 2010. Mobilized human hematopoietic stem/progenitor cells promote kidney repair after ischemia/reperfusion injury. Circulation, 121(20): 2211-2220 DOI 10.1161/CIRCULATIONAHA.109.928796.

28. Lin SL, Li B, Rao S, Yeo EJ, Hudson TE, Nowlin BT, Pei H, Chen L, Zheng JJ, Carroll TJ, Pollard JW, McMahon AP, Lang RA, Duffield JS. 2010. Macrophage Wnt7b is critical for kidney repair and regeneration. Proc Natl Acad Sci US A, 107(9): 4194-4199 DOI 10.1073/pnas.0912228107.

29. Lien YH, Lai LW, Silva AL. 2003. Pathogenesis of renal ischemia/reperfusion injury: lessons from knockout mice. Life Sci, 74(5): 543-552 DOI 10.1016/j.lfs.2003.08.001. 
369

370

371

372

373

374

375

376

377

378

379

380

381

382

383

384

385

386

387

388

30. Lorenzen JM, Kielstein JT, Hafer C, Gupta SK, Kümpers P, Faulhaber-Walter R, Haller H, Fliser D, Thum T. 2011. Circulating miR-210 predicts survival in critically ill patients with acute kidney injury. Clin J Am Soc Nephrol, 6(7): 1540-1546 DOI 10.2215/CJN.00430111.

31. Lu J, Kwan BC, Lai FM, Tam LS, Li EK, Chow KM, Wang G, Li PK, Szeto CC. 2010. Glomerular and tubulointerstitial miR-638, miR-198 and miR-146a expression in lupus nephritis. Nephrology (Carlton), 17(4): 346-351 DOI 10.1111/j.1440-1797.2012.01573.x.

32. Ma L, Qu L. 2013. The function of microRNAs in renal development and pathophysiology. J Genet Genomics, 40(4): 143-152 DOI 10.1016/j.jgg.2013.03.002.

33. Malek M, Nematbakhsh M. 2015. Renal ischemia/reperfusion injury; from pathophysiology to treatment. J Renal Inj Prev, 4(2): 20-27 DOI 10.12861/jrip.2015.06.

34. Mehta RL, Kellum JA, Shah SV, Molitoris BA, Ronco C, Warnock DG, Levin A; Acute Kidney Injury Network. 2007. Acute Kidney Injury Network: report of an initiative to improve outcomes in acute kidney injury. Crit Care, 11(2): R31 DOI 10.1186/cc5713.

35. Meng J, Zhang D, Pan N, Sun N, Wang Q, Fan J, Zhou P, Zhu W, Jiang L. 2015. Identification of miR-194-5p as a potential biomarker for postmenopausal osteoporosis. PeerJ, 3: e971 DOI 10.7717/peerj.971.

36. Mo L, Xiao X, Song S, Miao H, Liu S, Guo D, Li X, Bu C, Hou L, Yang X. 2015. Protective effect of Huang Gan formula in 5/6 nephrectomized rats by depressing the Wnt/ $\beta$-catenin signaling pathway. Drug Des Devel Ther, 9: 2867-2881 DOI 10.2147/DDDT.S81157.

37. Peng L, Ma J, Cui R, Chen X, Wei SY, Wei QJ, Li B. 2014. The calcineurin inhibitor tacrolimus 
389

390

391

392

393

394

395

396

397

398

399

400

401

402

403

404

405

406

407

408

reduces proteinuria in membranous nephropathy accompanied by a decrease in angiopoietin-like- 4 .

PLoS One, 9(8): e106164 DOI 10.1371/journal.pone.0106164.

38. Place RF, Li LC, Pookot D, Noonan EJ, Dahiya R. 2008. MicroRNA-373 induces expression of genes with complementary promoter sequences. Proc Natl Acad Sci U S A, 105(5): 1608-1613 DOI 10.1073/pnas.0707594105.

39. Saal S, Harvey SJ. 2009. MicroRNAs and the kidney: coming of age. Curr Opin Nephrol Hypertens, 18(4): 317-323 DOI 10.1097/MNH.0b013e32832c9da2.

40. Smoot ME, Ono K, Ruscheinski J, Wang PL, Ideker T. 2011. Cytoscape 2.8: new features for data integration and network visualization. Bioinformatics, 27(3): 431-432. DOI 10.1093/bioinformatics/btq675.

41. Srisawat N, Kellum JA. 2011. Acute kidney injury: definition, epidemiology, and outcome. Curr Opin Crit Care, 17(6): 548-555 DOI 10.1097/MCC.0b013e32834cd349.

42. Trionfini P, Benigni A, Remuzzi G. 2015. MicroRNAs in kidney physiology and disease. Nat Rev Nephrol, 11(1): 23-33 DOI 10.1038/nrneph.2014.202.

43. Waikar SS, Liu KD, Chertow GM. 2008. Diagnosis, epidemiology and outcomes of acute kidney injury. Clin J Am Soc Nephrol, 3(3): 844-861 DOI 10.2215/CJN.05191107.

44. Wang Y, Li L, Moore BT, Peng XH, Fang X, Lappe JM, Recker RR, Xiao P. 2012. MiR-133a in human circulating monocytes: a potential biomarker associated with postmenopausal osteoporosis. PLoS One, 7(4): e34641 DOI 10.1371/journal.pone.0034641.

45. Wei Q, Mi QS, Dong Z. 2013. The regulation and function of microRNAs in kidney diseases. IUBMB 
46. Wilflingseder J, Sunzenauer J, Toronyi E, Heinzel A, Kainz A, Mayer B, Perco P, Telkes G, Life, 65(7): 602-614 DOI 10.1002/iub.1174.

Langer RM, Oberbauer R. 2014. Molecular pathogenesis of post-transplant acute kidney injury: assessment of whole-genome mRNA and miRNA profiles. PLoS One, 9(8): e104164 DOI 10.1371/journal.pone.0104164.

47. Yu W, Wulf A, Liu T, Khoury MJ, Gwinn M. 2008. Gene Prospector: an evidence gateway for evaluating potential susceptibility genes and interacting risk factors for human diseases. $B M C$ Bioinformatics, 9: 528 DOI 10.1186/1471-2105-9-528.

\section{Figure legends}

Figure 1. IRI caused substantial changes in renal morphology and function. (A) Representative light microscopy images of HE-stained sections of renal outer medulla from the sham and IRI groups at 6, 12, 24 , and $48 \mathrm{~h}$. [magnification of the upper row, $200 \times($ bars $=250 \mu \mathrm{m})$; magnification of the lower row, $400 \times$ $($ bars $=200 \mu \mathrm{m})]$. White and black arrows indicate injured tubular and infiltrated inflammatory cells, respectively. (B) Representative light microscopy images of Masson-stained sections of renal outer medulla from the sham and IRI groups at 6, 12, 24, and $48 \mathrm{~h}$ (magnification, 100×; bars $=250 \mu \mathrm{m}$ ). (C) Graph showing the score of renal tubular injury in every group. $* * * p<0.0001$ versus sham; magnification, $200 \times$, $n=8$; (D) Serum creatinine levels at 6, 12, 24, and $48 \mathrm{~h}$ after unilateral IRI and in the sham group. $* * * p<$ 0.0001 versus sham; $n=8$.

Figure 2. Temporal correlation between the expression of global miRNAs and renal function indexes. (A) Temporal miRNA expression change within $48 \mathrm{~h}$ after renal IRI. miRNA abundance is defined as raw 
429

431

microarray signal output normalized to the blank value $0.1 ;{ }^{*} p<0.05$ versus sham (Kruskal-Wallis test).

(B and C) Temporal correlation between total miRNA abundance and index of tubulointerstitial injury/level of serum creatinine $(n=8)$. Total miRNA abundance is the sum of the abundance values of all miRNAs detected by microarray.

Figure 3. Temporal correlation between the expression of single miRNAs and renal function indexes. (A) Temporal expression changes of six miRNAs, including miR-18a, -34b, -134, -182, -210, and -214 $(n=8)$. $* p<0.05, * * p<0.001, * * * p<0.0001$ versus sham. (B and C) Temporal correlation between expression of individual miRNAs and the index of tubulointerstitial injury/level of serum creatinine $(n=8)$.

Figure 4. IRI induces release of inflammatory cytokines. (A) Representative immunofluorescence images of F4/80-labeled macrophages (green) from the sham and IRI groups at 6, 12, 24, and $48 \mathrm{~h}$. DAPI labels the nucleus (blue; Magnification, 200×; bars $=250 \mu \mathrm{m}$ ). (B) Graph indicating the number of macrophages in mice after sham or IRI. (C-E) Representative ELISA detection results for inflammatory markers (TNF$\alpha$, IL-1 $\beta$, and IL-6) in kidney tissue homogenates of IRI mice. ns: not significant versus sham; * $p<0.05$, ** $p<0.001, * * * p<0.0001$ versus sham; $n=8$.

Figure 5. IRI causes substantial PTC loss. (A) Representative images of mouse CD31-labeled outer medulla PTC post-ischemia/reperfusion injury kidneys at each time point (magnification, 200×; bars = 250 $\mu \mathrm{m})$. (B) Graph showing PTC loss for mice after IRI and sham. ns: not significant versus sham; ** $p<$ $0.001, * * * p<0.0001$ versus sham; $n=8$.

\section{Supplementary information}


449 Table S1. Primer sequences of genes used in this study (mouse).

450 Table S2. Significantly over-represented KEGG pathways in renal pathology.

451 Table S3. Renal pathology-related mRNAs targeted by miR-18a, $-134,-182,-210$, or -214 . 


\section{1}

Figure 1

IRI caused substantial changes in renal morphology and function. (A) Representative light microscopy images of HE-stained sections of renal outer medulla from the sham and IRI groups at $6,12,24$, and $48 \mathrm{~h}$. [magnification of the upper row, 200x (bars $=250 \mu \mathrm{m}$ ); magnification of the lower row, $400 \times($ bars $=200 \mu \mathrm{m}$ )]. White and black arrows indicate injured tubular and infiltrated inflammatory cells, respectively. (B) Representative light microscopy images of Masson-stained sections of renal outer medulla from the sham and IRI groups at 6, 12, 24, and $48 \mathrm{~h}$ (magnification, 100x; bars $=250 \mu \mathrm{m}$ ). (C) Graph showing the score of renal tubular injury in every group. ${ }^{* * *} p<0.0001$ versus sham; magnification, $200 x, n=8$; (D) Serum creatinine levels at 6, 12, 24, and $48 \mathrm{~h}$ after unilateral IRI and in the sham group. ${ }^{* * *} p<0.0001$ versus sham; $n=8$. 

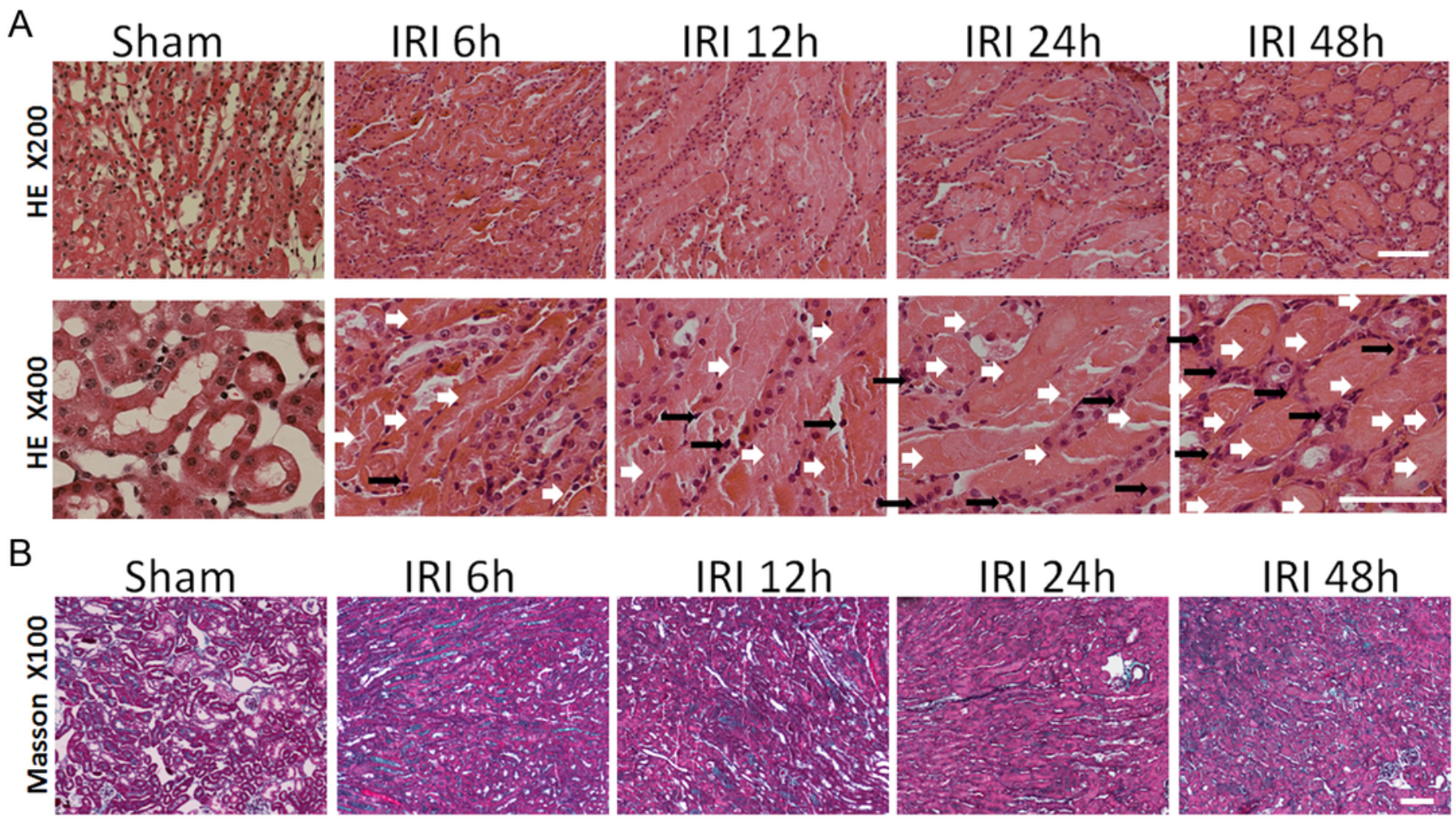

C

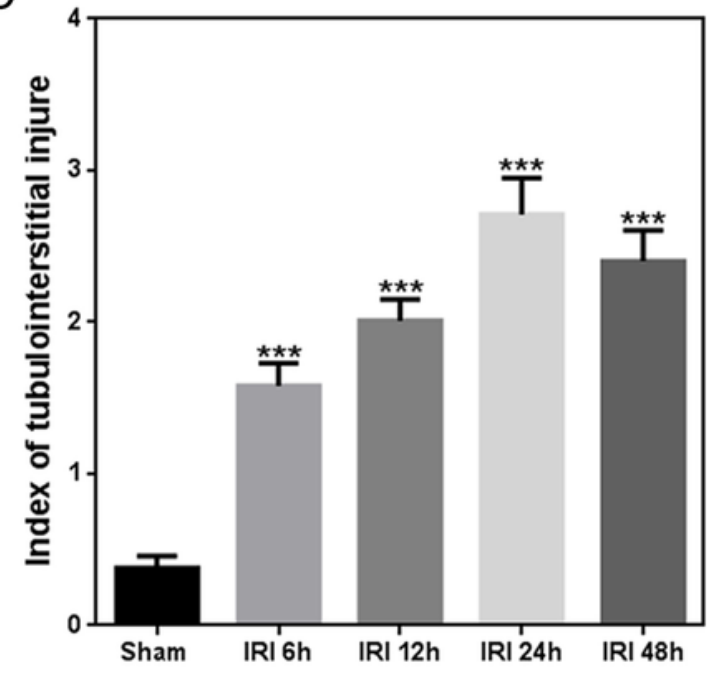

D

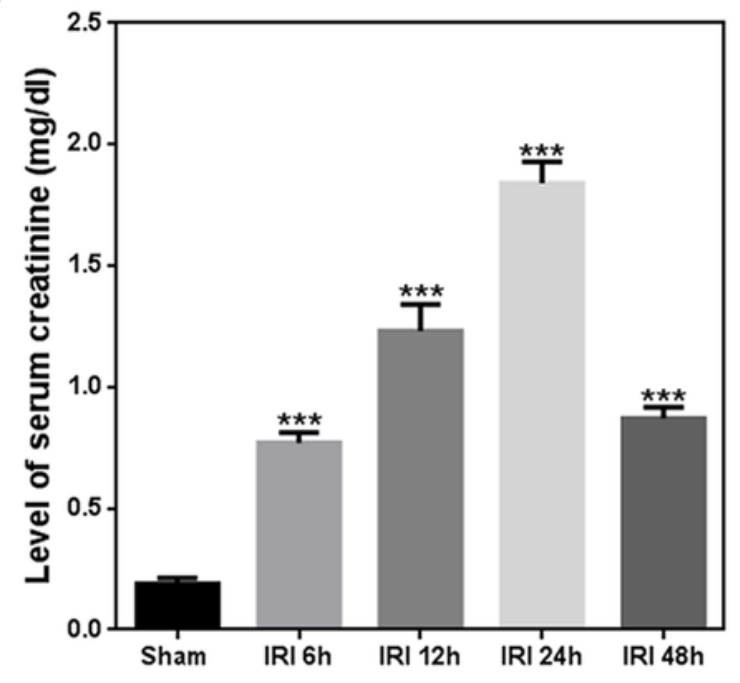




\section{2}

Figure 2

Temporal correlation between the expression of global miRNAs and renal function indexes.

(A) Temporal miRNA expression change within $48 \mathrm{~h}$ after renal IRI. miRNA abundance is defined as raw microarray signal output normalized to the blank value $0.1 ; *<0.05$ versus sham (Kruskal-Wallis test). (B and C) Temporal correlation between total miRNA abundance and index of tubulointerstitial injury/level of serum creatinine $(n=8)$. Total miRNA abundance is the sum of the abundance values of all miRNAs detected by microarray.

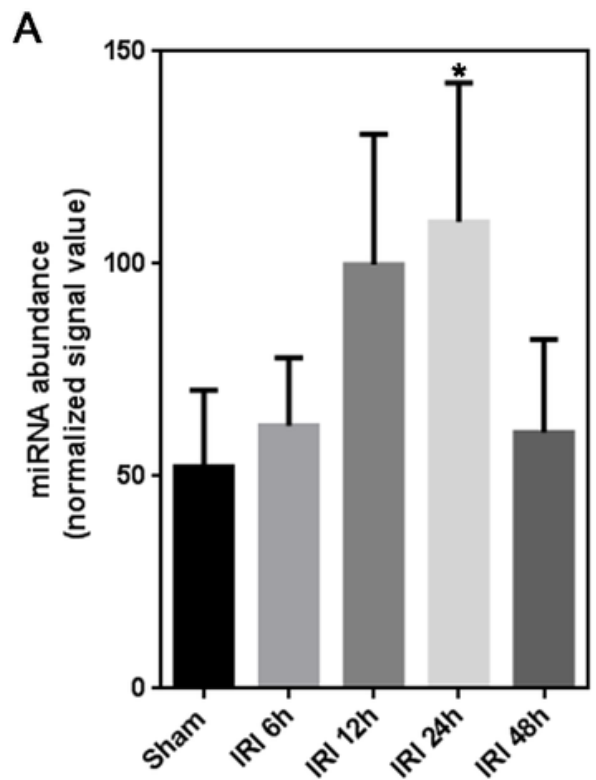

B

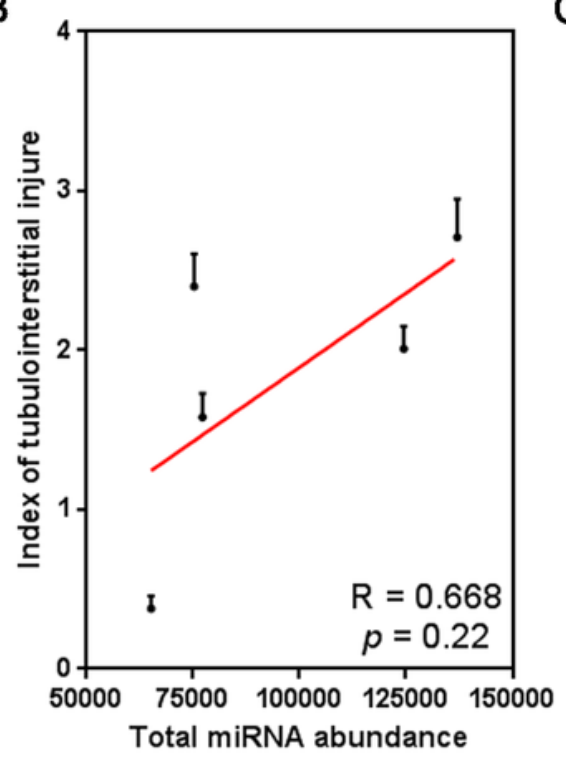

C

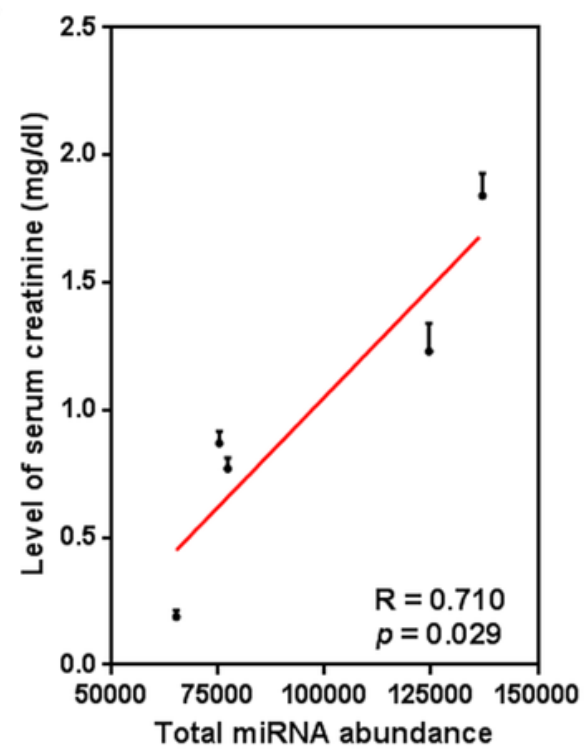




\section{3}

Figure 3

Temporal correlation between the expression of single miRNAs and renal function indexes.

(A) Temporal expression changes of six miRNAs, including miR-18a, -34b, -134, -182, -210, and -214 $(n=8) . * p<0.05, * * p<0.001$, *** $p<0.0001$ versus sham. (B and C) Temporal correlation between expression of individual miRNAs and the index of tubulointerstitial injury/level of serum creatinine $(n=8)$.

A
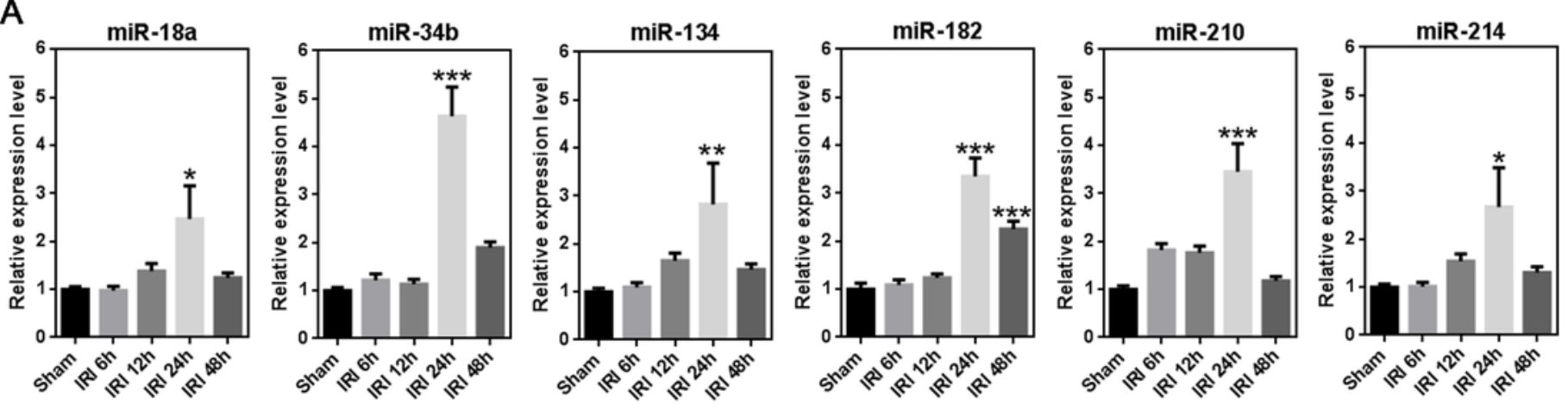

B
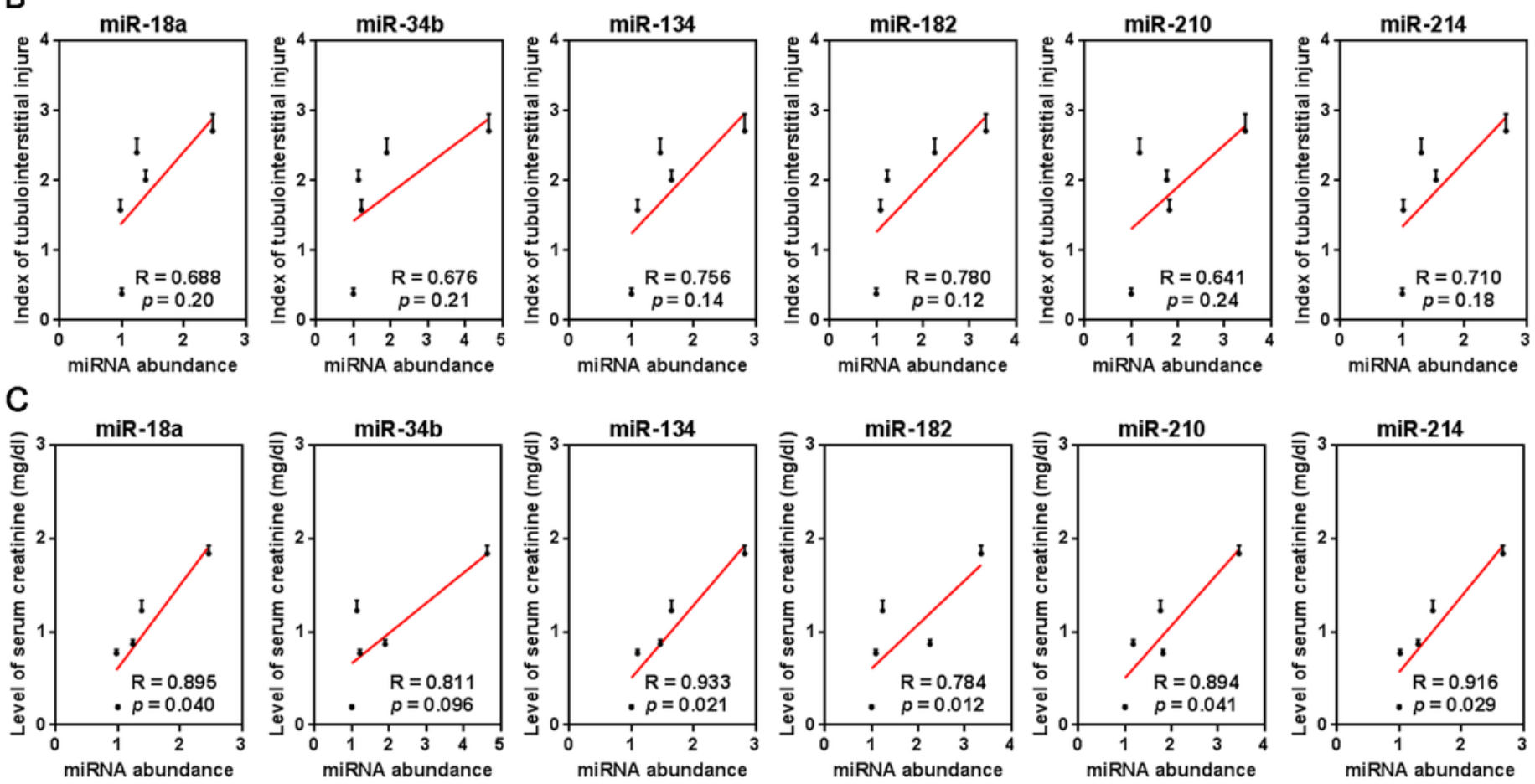


\section{4}

Figure 4

IRI induces release of inflammatory cytokines. (A) Representative immunofluorescence images of F4/80-labeled macrophages (green) from the sham and IRI groups at 6, 12, 24, and $48 \mathrm{~h}$. DAPI labels the nucleus (blue; Magnification, 200x; bars $=250 \mu \mathrm{m}$ ). (B) Graph indicating the number of macrophages in mice after sham or IRI. (C-E) Representative ELISA detection results for inflammatory markers (TNF- $\alpha$, IL-1 $\beta$, and IL-6) in kidney tissue homogenates of IRI mice. ns: not significant versus sham; $* p<0.05$, ** $p<0.001$, *** $p<$ 0.0001 versus sham; $n=8$. 


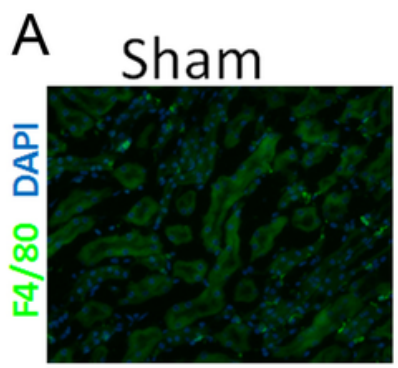

B

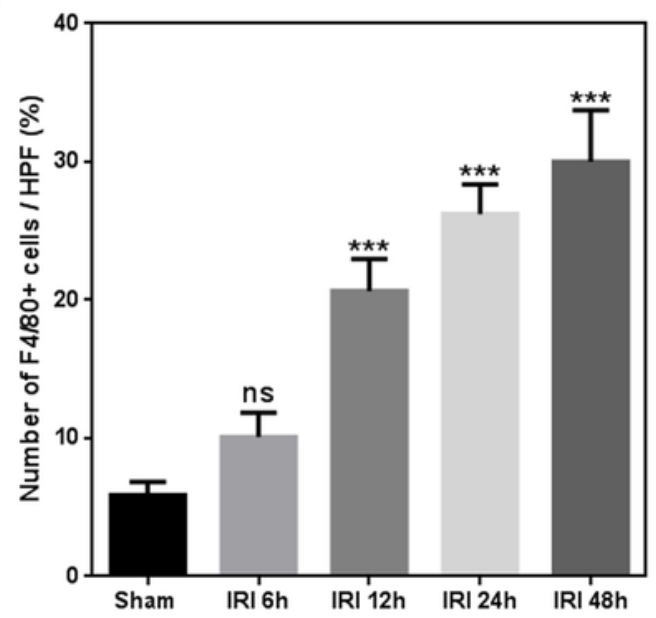

D

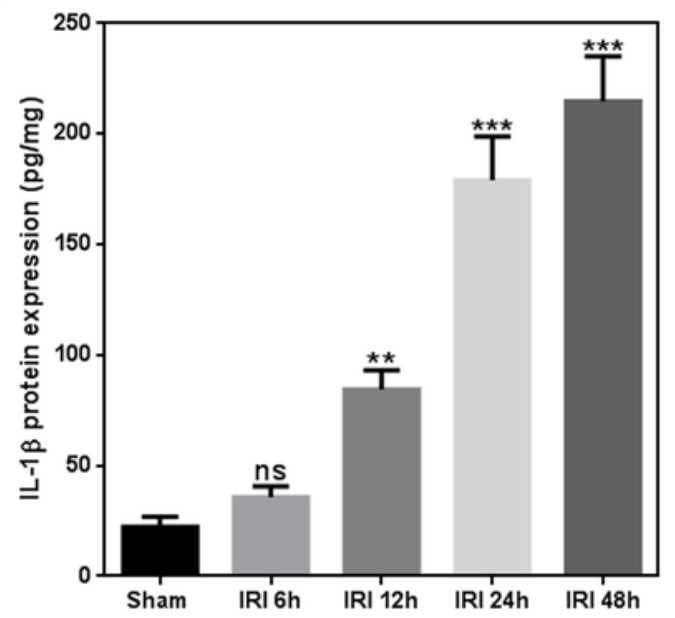

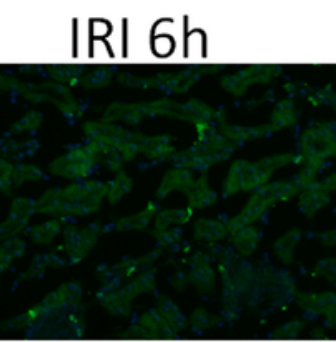
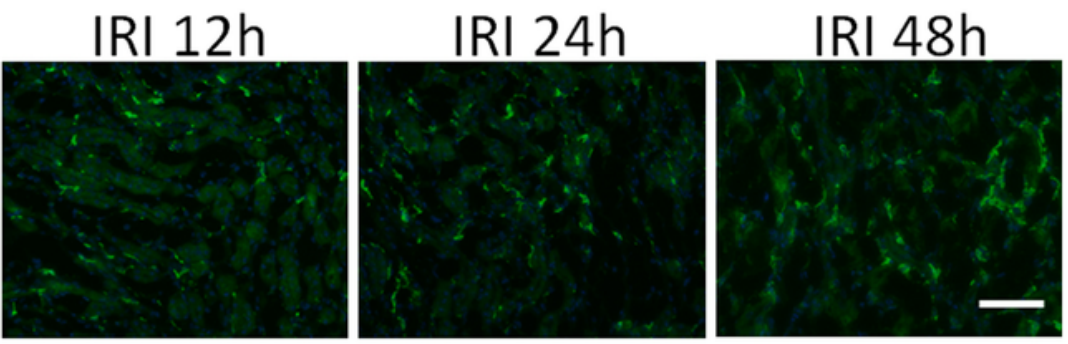

\section{C}

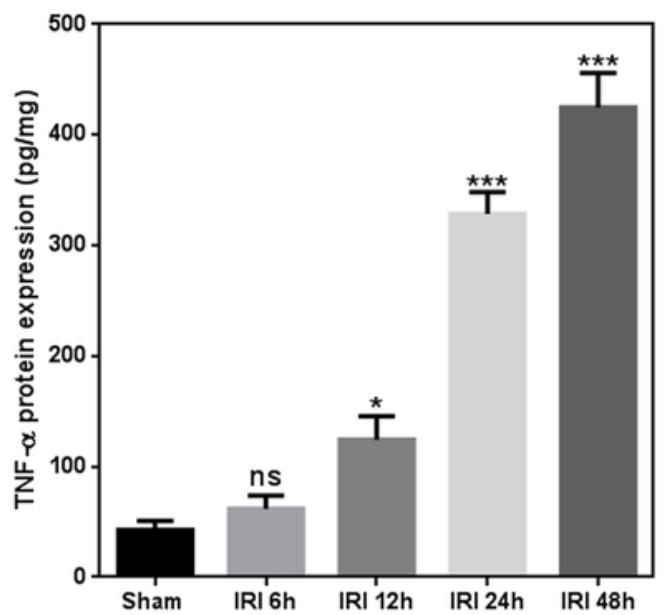

$E$

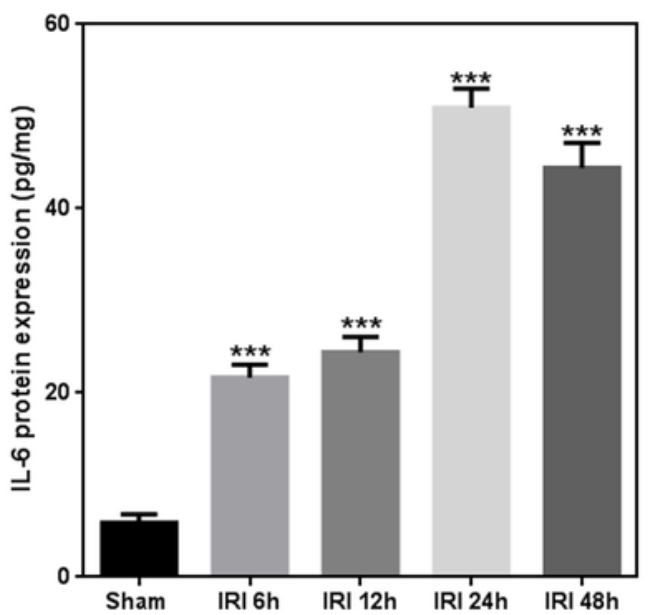




\section{5}

Figure 5

IRI causes substantial PTC loss. (A) Representative images of mouse CD31-labeled outer medulla PTC post-ischemia/reperfusion injury kidneys at each time point (magnification, 200x; bars $=250 \mu \mathrm{m}$ ). (B) Graph showing PTC loss for mice after IRI and sham. ns: not significant versus sham; ** $p<0.001, * * * p<0.0001$ versus sham; $n=8$.

A
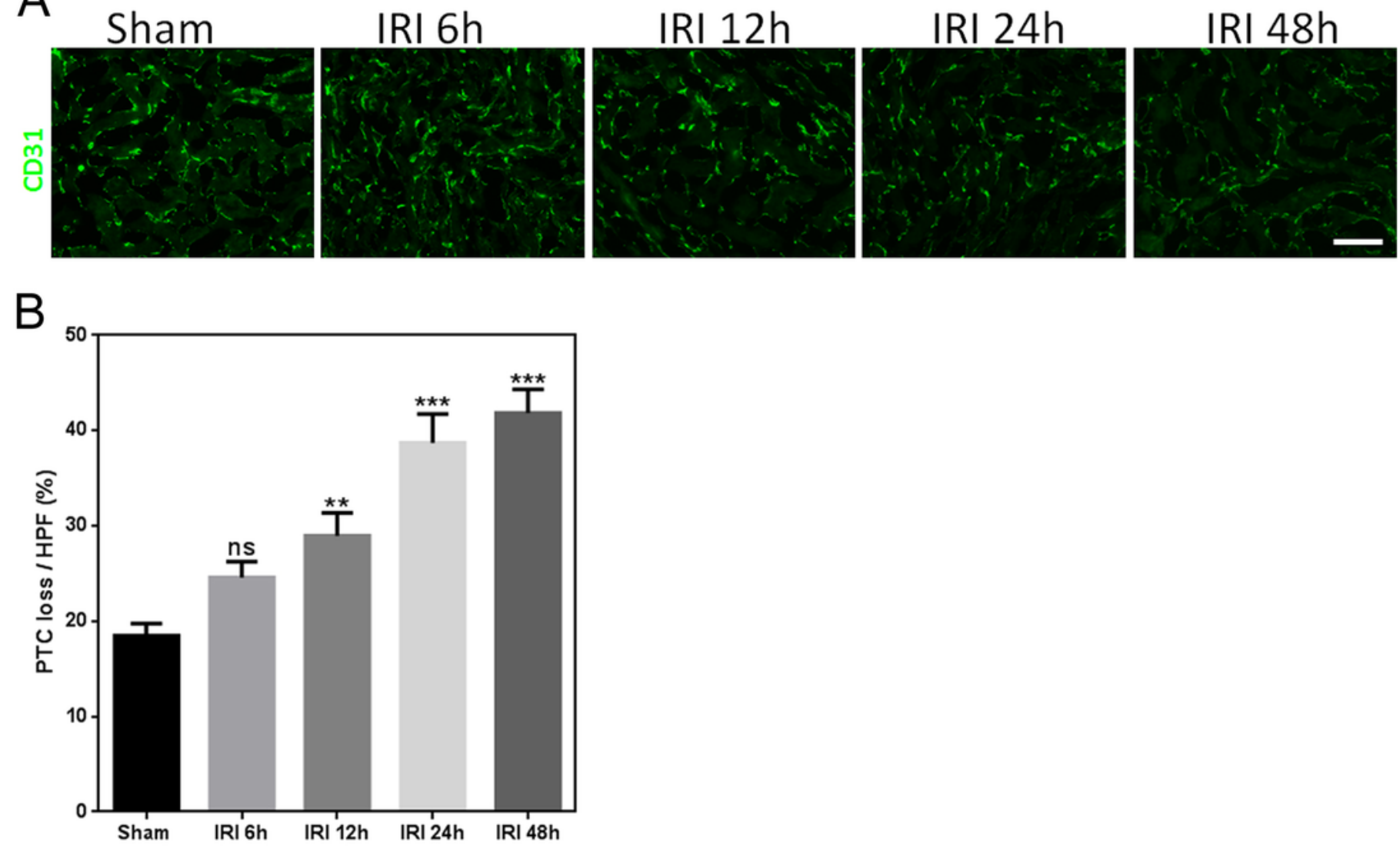